\title{
Il contributo allo sviluppo della professione infermieristica delle congregazioni religiose del XIX secolo
}

\author{
Contribution of 19th-century religious congregations in the development of the nursing profession
}

Sabrina Bezze ${ }^{1}$

Edoardo Manzoni2

Stefania Di Mauro ${ }^{3}$

\section{RIASSUNTO}

Lo scopo del presente lavoro di ricerca storica è di esaminare il contributo allo sviluppo della professione infermieristica delle congregazioni religiose del XIX secolo prendendo come esempio le Suore di Maria Bambina conosciute anche come Suore della Carità delle Sante Bartolomea Capitanio e Vincenza Gerosa.

Sono state analizzate 63 fonti bibliografiche reperite nell'archivio storico Casa Madre delle Suore di Maria Bambina a Milano in via S. Sofia n.13, ad esclusione di una fonte reperita presso la Biblioteca Nazionale Braidense di Milano. Il lavoro di ricerca ha posto una lente d'ingrandimento sul contributo che le Suore di Maria Bambina hanno fornito, rispetto alle aree professionali e allo sviluppo degli attributi distintivi della professione infermieristica nell'ottica sociologica (Greenwood, 1980).

Per l'analisi delle fonti documentarie è stato applicato il metodo di ricerca storica secondo Chabod (2006). Per l'esegesi delle fonti si è creata una griglia di analisi della fonte documentaria.

Dall'aggregazione dei dati sono emersi due filoni d'indagine: il primo riguarda il ruolo di Suor Emilia Vinante come esperta per la professione infermieristica e il secondo per quanto attiene alla politica professionale promossa dalla Federazione Italiana Religiose Ospedaliere ( FIRO).

Alla luce dei risultati della ricerca si può affermare che le congregazioni religiose hanno operato nell'ambito della professione infermieristica diventando una forza sociale in grado di incidere in modo determinante sullo sviluppo culturale e professionale degli infermieri.

Parole Chiave: formazione, storia dell'assistenza infermieristica, infermiere religiose

ABSTRACT

The purpose of this historical research project is to examine the contribution of 19th-century religious congregations in the development of the nursing profession, based on the historical example of the Sisters of Charity of Sts. Bartolomea Capitanio and Vincenza Gerosa (or the Sisters of the Holy Child Mary).

To this end, sixty three volumes were analysed, all taken from the historical archive of the Generalate of the Sisters of the Holy Child Mary in Milan, in via S. Sofia n.13, with the exception of just one, taken from the Braidense National Library, also in Milan.

This research project has highlighted the sociological contribution provided by the Sisters of the Holy Child Mary to the professional nature of nursing, and to the development of the distinctive features of the nursing profession (Greenwood, 1980).

All documentary sources were analysed in line with the Chabod historical research method (2006), and for their critical interpretation, a scheme of analysis was created.

Two lines of investigation emerged from the data collected: the role of Sister Emilia Vinante as an expert with regard to the nursing profession, and the professional strategies promoted by FIRO (Federation of Italian Religious Nurses).

Based on the conclusions of the research project, it may be stated that religious congregations contributed greatly to the nursing profession, leaving a decisive mark on the cultural and professional development of nurses.

Key words: nursing education, historical research, religiuos nurse

INTRODUZIONE

$\mathrm{L}$ a scelta dell'argomento di ricerca storica, nasce dall'esperienza personale di formazione durante la scuola per infermieri professionali diretta da religiose negli anni 1980-83, dalla percezione che la convivenza con le suore abbia influito profondamente nella cultura

1 Coordinatore didattico corso di laurea in infermieristica UNIMIB, IRCCS Multimedica. Corrispondenza: sabrina.bezze@multimedica.it

2 Direttore Generale, Istituto Palazzolo Bergamo

3 Prof. Associato, Università degli Studi di Milano Bicocca, Facoltà di Medicina e Chirurgia. infermieristica e che questa influenza sia di portata superiore rispetto alla nostra conoscenza attuale.

Un altro fattore d'interesse è emerso dalla ricerca di testi in merito alle congregazioni religiose e dalla successiva scoperta della scarsa attenzione da parte degli storici rispetto al fenomeno oggetto d'indagine. Rocca (1992) segnala che nella recente storiografia della donna italiana le religiose non sono quasi mai considerate. Colombo (2004) rileva un risveglio d'interesse per lo studio delle congregazioni religiose, ma alla quale non soccorre alcuna tradizione di ricerca consolidata, né in campo storico economico, né in quello della storia reli- 
giosa in età contemporanea, né nella storiografia prodotta dalle stesse congregazioni, ancora troppo caratterizzate da impostazioni prevalentemente agiografiche. Bartoloni (2007) in un recente studio tra le donne laiche e religiose tra ottocento e novecento afferma che, in effetti, sulle congregazioni femminili poco si aveva a disposizione ad eccezione di una rapida notazione di Giacomo Martina e degli studi di Giancarlo Rocca.

La nascita e lo sviluppo delle congregazioni religiose, affermatosi e consolidatosi nella seconda metà dell'Ottocento europeo, furono un fenomeno di proporzioni imponenti: si assistette in Europa alla nascita e all'espansione di centinaia d'istituti di vita comune, soprattutto femminili di voti semplici.

La nascita della congregazione religiosa ha legittimato la donna nella carica di superiora generale di un istituto propriamente religioso e le ha permesso di proporsi come persona in grado di aiutare a risolvere i problemi educativo-assistenziali-sociali del proprio tempo (Rocca, 1992).

Gli ambiti d'impegno delle congregazioni religiose, dopo una prima fase di risposta alle esigenze della popolazione, si allargarono e si differenziano in tipologie e sistemi d'intervento in relazione al carisma del fondatore dell' istituto, mentre l'elemento comune delle congregazioni rimase l'assistenza e la formazione (Colombo, 2004).

La ragione per cui molte delle congregazioni religiose furono chiamate a gestire interi nosocomi, sta in diversi fattori: era diffusa una certa mentalità che prediligeva la donna come infermiera, migliore ancora se nubile; la serietà, il rigore morale delle suore e l'economicità delle loro prestazioni; non da ultimo le religiose erano disposte a curare il malato anche in caso di contagio ed erano disponibili all'assistenza sia di giorno sia di notte (Manzoni, 2005).

Il presupposto che ha guidato il lavoro di ricerca è che la storiografia femminista e lo stimolo alla modernizzazione e laicizzazione del paese, abbiano oscurato il contributo che le congregazioni religiose hanno dato alla professione infermieristica. La presente ricerca storica riguarda le suore della Carità delle Sante Bartolomea Capitanio e Vincenza Gerosa, conosciute anche come Suore di Carità di Lovere o Suore di Maria Bambina, in quanto fu una delle congregazioni lombarde e italiane di maggior rilievo sia per la diffusione e il numero di membri sia per il contributo che diedero allo sviluppo dell'assistenza infermieristica in ospedale e alla formazione delle allieve nelle scuole convitto per infermiere. (Carraro, Mascotti, 1987).

Scopo di questo lavoro, è stato di individuare attraverso la descrizione, l'analisi e l'interpretazione delle fonti documentarie oggetto di studio, il contributo che le Suore di Maria Bambina hanno dato alla professione infermieristica.

Gli obiettivi specifici individuati sono: evidenziare attraverso lo studio delle fonti documentarie, il contributo delle Suore di Maria Bambina rispetto alle principali aree professionali: assistenza diretta, organizzazione/amministrazione, formazione.

evidenziare attraverso lo studio delle fonti documentarie, il contributo che le Suore di Maria Bambina hanno dato allo sviluppo degli attributi distintivi della professione infermieristica, nell'ottica sociologica.

\section{MATERIALI E METODI}

La metodologia per il reperimento delle fonti si è basata su una presa di contatto iniziale con le storiografe dell'archivio storico Casa Madre delle Suore di Maria Bambina a Milano in via S. Sofia n.13. Per accertare la presenza di materiale documentario, sono stati compiuti successivi sopralluoghi per la ricognizione e scelta delle fonti da inserire nello studio. Tutti i documenti analizzati sono stati reperiti presso il medesimo archivio storico ad eccezione del manuale di Suor Emilia Vinante che è avvenuto presso la Biblioteca Nazionale Braidense di Milano. La scoperta del testo di Vinante è avvenuta consultando la bibliografia del lavoro di Sironi (1992).

In seguito ad una ricerca online tramite il Sistema Bibliotecario Nazionale, è stato possibile trovare alcune edizioni del manuale, tra cui la prima, conservata nella biblioteca Nazionale Centrale di Firenze.

I documenti da includere nello studio sono stati scelti riguardo ai seguenti elementi:

1) periodo storico considerato (1896-1980): nell'anno 1896, a Napoli, iniziava la sua attività la scuola della Croce Azzurra, prima scuola per infermiere aperta in Italia secondo il sistema Nightingale per iniziativa della principessa Adelaide Pignatelli che ne affidò la direzione a Grace Baxter, un'infermiera italoamericana che aveva operato in uno degli ospedali americani più prestigiosi. Nell'anno 1980, inizia il graduale ritiro delle congregazioni religiose dagli ospedali pubblici.

2) Tipologia delle fonti:

- Convenzioni;

- Corrispondenze tra la superiora della casa generalizia, la superiora locale, l'amministrazione ospedaliera;

- Relazioni, quaderni, corsi di formazione.

Le fonti reperite (vedi tabella I), sono state collocate in ordine cronologico all'interno dei seguenti periodi storici: il governo fascista e gli anni della seconda guerra mondiale (1925-1945), il secondo dopoguerra, (19461952), il periodo della costruzione della repubblica (1953-1964) e i primi anni 60 e gli anni di piombo (1965-1980). 


\begin{tabular}{|c|c|}
\hline Tipologia & Numero \\
\hline Lettere & 21 \\
\hline Relazioni & 11 \\
\hline Libro & 1 \\
\hline Editoriali & 3 \\
\hline Articoli & 2 \\
\hline Programmi di corsi di formazione & 2 \\
\hline Riviste & 23 \\
\hline Totale & 63 \\
\hline
\end{tabular}

Tabella I - Tipologia e numero di fonti documentarie incluse nello studio.

Per l'analisi delle fonti documentarie è stato applicato il metodo di ricerca storica secondo Chabod (2006) che prevede l'analisi filologica che consiste nella descrizione materiale della fonte e nella dimostrazione di autenticità formale; e la parte interpretativa che consiste nella interpretazione delle fonti in relazione agli obiettivi specifici. Per l'esegesi delle fonti si è creata una griglia d'analisi.

La prima sezione analizza la tipologia della fonte e le caratteristiche materiali, la seconda dimostra l'autenticità attraverso l'esame estrinseco ed intrinseco, la terza sezione analizza le aree professionali e gli attributi distintivi professionali. La griglia è consultabile nell'allegato 1 .

Le 40 fonti documentarie sono state analizzate con la griglia d'analisi. Per quanto riguarda i numeri delle 23 riviste sono stati analizzati gli argomenti degli indici della rivista "Quaderni FIRO" per un totale di 137 articoli.

L'analisi interpretativa si basa sugli obiettivi specifici di codesta ricerca storica.

\section{RISULTATI}

La professione infermieristica è stata sviluppata non solo dalla componente laica ma anche dalla componente religiosa femminile. Per coronare questa ipotesi si è preso come esempio, la congregazione delle due sante Bartolomea Capitanio e Vincenza Gerosa, dall'analisi dei documenti è emerso che la congregazione ha agito attraverso le sue suore, in particolare si è distinta Suor Emilia Vinante ma soprattutto attraverso una politica professionale promossa dalla Federazione Italiana Religiose Ospedaliere (F.I.R.O). Questa federazione nasce nel 1949, quando Pio XII costituisce la "Commissione Pontificia per le Religiose Ospedaliere" nominando primo presidente il Cardinale Urbani. La commissione promuove la costituzione della FIRO che inizia ad agire sotto la guida della Commissione Pontificia, ne sviluppa la vita e le attività allo scopo di promuovere un vasto movimento di qualificazione professionale delle attività dei singoli istituti religiosi e tutelare la loro opera negli stessi ospedali dove da secoli erano testimoni della carità della chiesa. Le Suore di Maria Bambina furono direttamente coinvolte nella associazione attraverso l'elezione di Suor Emilia Vinante, direttrice della scuola convitto per infermiere professionali di Niguarda dal 1935 al 1968, in qualità di prima presidente della F.I.RO dal 1949 al 1953 e di Suor Costantina Baldinucci, superiora generale delle Suore di Maria Bambina dal 1957 al 1969 che è stata presidente della FIRO dal 1963 al 1968.

L'analisi dei documenti si muove tra 2 filoni d'indagini: il ruolo di esperta esercitato da Vinante (vedi tabella II) e la politica professionale promossa dalla FIRO (vedi tabella III).

\begin{tabular}{|c|c|}
\hline Tipologia & Numero \\
\hline Relazioni di Suor Emilia Vinante & 3 \\
\hline Testo di Suor Emilia Vinante & 1 \\
\hline $\begin{array}{c}\text { Relazioni di G. Sollazzo, M. Sforza, sulla scuola professiona- } \\
\text { le convitto infermiere per religiose e laiche degli Istituti } \\
\text { Ospitalieri di Milano }\end{array}$ & 2 \\
\hline $\begin{array}{l}\text { Lettere di richiesta di partecipazione a suor Vinante a due } \\
\text { convegni e ad un incontro del consiglio dell'Associazione } \\
\text { Provinciale I.P. e A.S.V. }\end{array}$ & 3 \\
\hline $\begin{array}{l}\text { Relazione inviata da Maria Sforza alla superiora delle Suore } \\
\text { di Maria Bambina sull'organizzazione di una scuola convit- } \\
\text { to cattolica inglese }\end{array}$ & 1 \\
\hline Testo di Suor Emilia Vinante & 1 \\
\hline $\begin{array}{l}\text { Preghiera delle allieve della scuola convitto infermiere } \\
\text { degli istituti ospitalieri di Milano }\end{array}$ & 1 \\
\hline Totale & 12 \\
\hline
\end{tabular}

\begin{tabular}{|c|c|}
\hline Tipologia & Numero \\
\hline Lettere/programmi di giornate di studio & 9 \\
\hline Articoli “Quaderni F.I.R.O.” & 5 \\
\hline $\begin{array}{l}\text { Lettere richiesta di suore da parte della FIRO per le ammini- } \\
\text { strazioni ospedaliere }\end{array}$ & 3 \\
\hline Lettere cambi/nomine presidenza FIRO & 4 \\
\hline $\begin{array}{l}\text { Relazioni: } 2 \text { convenzione enti ospedalieri, } 1 \text { elenco scuole } \\
\text { convitto dirette da religiose, } 1 \text { presentazione del } 3^{\circ} \text { conve- } \\
\text { gno della FIRO }\end{array}$ & 4 \\
\hline Lettera di commento ad un articolo anticlericale & 1 \\
\hline Lettera con la relazione della statistica delle diplomate & 1 \\
\hline $\begin{array}{c}\text { Lettera della S. Congregazione dei religiosi, strategie d'azione } \\
\text { in previsione dell'applicazione della legge Mariotti }\end{array}$ & 1 \\
\hline Quaderni F.I.R.O. & 23 \\
\hline Totale & 51 \\
\hline
\end{tabular}




\section{Suor Emilia Vinante}

Eugenia Vinante nasce a Tesero nel 1908. A vent'anni espresse la sua vocazione ed entrò a Trento nella Congregazione delle Suore di Maria Bambina, scegliendo di chiamarsi con il nome del padre, Emilio.

Nel dicembre 1930 frequenta la Scuola Convitto Infermiere appena istituita presso gli Istituti Ospitalieri di Milano (l'attuale Ospedale Maggiore in via Francesco Sforza).

Questa scuola divenne in seguito modello per altre in Italia, (Carraro, Mascotti 1987).

Si diplomò infermiera professionale e conseguì l'abilitazione di capo sala. Iniziò la sua attività presso la Scuola, operando come capo sala didattica.

Suor Emilia Vinante, si distinse per un brillante curriculum scolastico e per le sue doti di relazione e di organizzazione intelligente, la contessa Maria Sforza prima direttrice la volle sua vicedirettrice nel 1935, e dopo venti giorni di prova le lasciò la direzione della scuola, (Carraro, Mascotti 1987).

Dopo pochi anni, nel 1939, la Scuola si trasferì presso il nuovo ospedale di Niguarda, dove era stato edificato un intero padiglione per la Scuola ed il Convitto, costruito secondo i più moderni criteri, per accogliere 150 allieve.

Negli oltre trent'anni di direzione di Suor Vinante vennero diplomate 2205 infermiere professionali e promosse 313 capo sala abilitate a funzioni direttive.

Suor Emilia Vinante scrisse nel 1949, la prima edizione del testo: "Note di tecnica e pratica per l'assistenza infermieristica medico-chirurgica", il formato tascabile era pensato affinché le allieve lo portassero con sé durante le attività di reparto. La consegna del manuale, nelle sue varie edizioni è avvenuta dal 1949 al 1987.

Suor Vinante introdusse nel 1947 il corso di psicologia, di particolare utilità per conoscere la psicologia dell'infermo, le reazioni causate dall'evento malattia o provocate dall'ambiente ospedaliero, (Sollazzo, 1956), creò inoltre corsi di specializzazione per infermiere professionali in assistenza chirurgica (1947), in assistenza pediatrica (1953).

Il contributo fondamentale di Suor Vinante è la creazione di un corso per Aspiranti Direttrici di Scuole Infermiere organizzato in collaborazione con l'Istituto Giuseppe Toniolo di Studi Superiori ente fondatore e finanziatore dell'Università Cattolica del Sacro Cuore e la Scuola Convitto per infermiere professionali degli Istituti Ospitalieri di Milano, dall'anno 1953 all'anno 1965 (Negri, 2005).

Questo corso che si proponeva di formare direttrici e vicedirettrici per la direzione delle Scuole Convitto, ha colmato una delle maggiori lacune dell'insegnamento infermieristico italiano. (Sollazzo, 1956).
Lo studio e l'analisi delle relazioni di Vinante (1949, 1954) rivelano una donna di straordinaria intelligenza, molto attenta ai problemi reali della professione. Le fonti documentarie (Senni, 1946, Campi, 1948) dimostrano che Suor Vinante era un'esperta, un punto di riferimento per la professione infermieristica, partecipò alle commissioni di studio per i problemi infermieristici presso il Ministero della Sanità, (Reali,1948) e fu eletta prima presidente della F.I.R.O. nel 1949.

L'opera di Suor Vinante venne riconosciuta pubblicamente nel 1963 con il conferimento della medaglia di bronzo al merito della Sanità Pubblica e nel 1968 con la medaglia d'oro del Collegio delle Infermiere Professionali, (Negri, 2007).

\section{Federazione Italiana Religiose Ospedaliere (FIRO)}

Il secondo filone d'indagine riguarda la F.I.RO. I campi di attività della FIRO sono le relazioni con l'esterno e la presenza presso gli organi legislativi ed esecutivi per suggerire e tutelare il patrimonio della chiesa in campo assistenziale.

Essa partecipa alle commissioni di studio per i problemi infermieristici presso il Ministero della Sanità, alla gestione e alla vita dei collegi provinciali IP-ASVVI, alla preparazione delle convenzioni con le Regioni e le USL preoccupandosi della posizione giuridica ed economica delle suore infermiere (Cosseta, 1994).

Nel campo professionale il primo atto di fondamentale importanza della FIRO è di promuovere l'applicazione della legge istitutiva delle Scuole Convitto per Infermiere Professionali e per Assistenti Sanitarie (RDL 15 agosto 1925 n. 1832, e relativo RD 21 novembre1929 n.2330). Da allora essa si è fatta promotrice dell'istituzione di scuole per infermieri professionali destinate sia a religiose sia a laiche, ritenendo che la preparazione di personale qualificato per l'assistenza è un dovere per le comunità religiose che si dedicano all'assistenza infermieristica. Ciò è avvenuto spesso con mezzi patrimoniali propri delle congregazioni (ad esempio le scuole per infermiere professionali di Ivrea e di Arezzo) (Cosseta, 1994). Nella relazione della FIRO (1963) sono elencate 67 scuole convitto dirette da religiose. Nella tabella IV si riportano l'elenco delle Scuole Convitto dirette dalle Suore di Maria Bambina mentre nella tabella $\mathrm{V}$ è possibile visionare l'elenco delle altre congregazioni che dirigono le scuole convitto per infermiere professionali.

La FIRO ha indetto Convegni e organizzato corsi d'aggiornamento per dirigenti e docenti di scuole e servizi infermieristici, superiori di ospedali, infermiere, delegate FIRO e allieve. Sono 34 i corsi individuati dai documenti analizzati, sottoforma di programma delle giornate di studio, di inviti alle madri generali delle congregazioni religiose appartenenti alla FIRO o come comunicazioni presenti nel notiziario dei Quaderni 


\begin{tabular}{|c|c|}
\hline Scuole convitto per Infermiere Professionali & Città \\
\hline Scuola convitto per I. P, presso I'ospedale Maggiore & BERGAMO \\
\hline Scuola convitto per I. P, presso l'ospedale Civile & COMO \\
\hline $\begin{array}{c}\text { Scuola convitto per I.P., presso I'Ospedale Maggiore } \\
\text { "Niguarda", }\end{array}$ & MILANO \\
\hline $\begin{array}{c}\text { Scuola convitto per I.P., presso Istituti Clinici di } \\
\text { Perfezionamento, }\end{array}$ & MILANO \\
\hline $\begin{array}{c}\text { Scuola convitto per I.P., presso La casa di cura } \\
\text { "Capitanio", }\end{array}$ & MILANO \\
\hline $\begin{array}{c}\text { Scuola convitto per I.P., presso Ospedale Gerardo dei } \\
\text { Tintori, }\end{array}$ & MONZA \\
\hline $\begin{array}{c}\text { Scuola convitto per Vigilatrici d'Infanzia, presso Istituto } \\
\text { Prov.le Assistenza Infantile }\end{array}$ & MONZA \\
\hline $\begin{array}{c}\text { Scuola convitto per I.P., presso La Facoltà di Medicina } \\
\text { dell'Università Cattolica del S. Cuore. }\end{array}$ & ROMA \\
\hline $\begin{array}{c}\text { Scuola convitto per I.P., presso I'Istituto S: Corona. } \\
\text { SASSARI }\end{array}$ \\
\hline
\end{tabular}

Tabella IV Elenco delle Scuole Convitto per I.P dirette dalle Suore di Maria Bambina nel 1963.

\begin{tabular}{|c|}
\hline Altre Congregazioni Religiose \\
\hline Suore della Carità della S.C.A. Thouret \\
\hline Figlie della Carità di S. Vincenzo de' Paoli \\
\hline Missionarie Zelatrici del S. Cuore \\
\hline Piccole Suore della Sacra Famiglia \\
\hline Minime dell'addolorata \\
\hline Suore della Carità dell'Immacolata Concezione \\
\hline Figlie di S. Anna \\
\hline Ancelle della Carità \\
\hline Figlie della Sapienza \\
\hline Suore di NS del Rifugio in Monte Calvario \\
\hline Suore della Provvidenza \\
\hline Suore di SG Cottolengo \\
\hline Figlie del cuore di Maria \\
\hline Francescane Missionarie di Maria \\
\hline Suore Maestre di S Dorotea Figlie dei SS Cuori \\
\hline Suore francescane Elisabettine \\
\hline Suore Francescane di Susa \\
\hline Sorella Misericordia \\
\hline
\end{tabular}

Tabella V Elenco delle altre Congregazioni Religiose che dirigono le scuole convitto per infermiere professionali nel 1963.

FIRO I destinatari dei corsi, sono suore, superiore di ospedali, direttrici di scuole convitto per I.P., A.S.V., V.I., infermiere religiose e laiche. I corsi sono stati organizzati nell'arco di tempo dal 1954 al 1967, nelle seguenti località: Novara, Roma, Pallanza, Como, Brindisi, Loreto, Cagliari, Selva di Fasano, Milano, Napoli, Trento, La Verna, Castenuovo Fogliani, Venezia,
Padova, Torino, Palermo, Parma, Cagliari. Tra le varie attività e gli impegni assunti, la FIRO per rispondere alle esigenze associative e per le necessità di aggiornamento redige una rivista propria "Quaderni F.I.R.O", (Cosseta, 1994). I quaderni analizzati sono 23, il primo numero "Quaderni FIRO" "Infirmus eram et visitastis me" è dell'anno $1^{\circ}$ N.1, gennaio-febbraio-marzo 1956, l'ultimo "Quaderni FIRO delle religiose ospedaliere" è dell'anno XIII Num. 2 marzo aprile 1968. Non è stato possibile, in quanto mancanti analizzare i numeri della rivista dall'anno 1962 all'anno 1964.

Padre Molinari (1956) spiega lo scopo della rivista FIRO: 'Il rapido sviluppo delle scienze sanitarie, il perfezionamento delle istituzioni sanitarie civili di questi ultimi decenni, richiedono con urgenza, l'aggiornamento tecnico professionale di tutte le religiose infermiere, il diploma di stato, infatti, oltre a conferire loro un diritto, impone anche il dovere di acquistare quelle conoscenze di carattere scientifico, che mettono ogni diplomata in condizione di dare la propria opera nel modo più assoluto e perfetto possibile. Nella parte introduttiva dell'editoriale Padre Molinari (1956) propone un'analisi sull'aggiornamento dell'infermiera professionale che apprende dalle colleghe e dai medici, ma non ha il tempo e i testi a disposizione per aumentare le proprie conoscenze medico-chirurgiche. Padre Molinari pur considerando l'impegno gravoso delle suore nelle corsie è intransigente rispetto alla preparazione tecnico-professionale.

Quali erano le necessità di aggiornamento delle suore, in che ambito e quali erano gli argomenti più affrontati?

Per rispondere a questa domanda sono stati analizzati gli articoli della rivista "Quaderni FIRO".

Le riviste analizzate sono 23, gli articoli 143 . Non sono stati considerati nella catalogazione gli editoriali e le comunicazioni finali che riguardano la vita della Federazione. La catalogazione è avvenuta leggendo i titoli degli articoli dell'indice di ogni quaderno; nel caso in cui il titolo non era indicativo dell'argomento, si è proceduto a una lettura sommaria dell'articolo.

Gli articoli sono stati raggruppati in argomenti, secondo la suddivisione proposta da Cossetta (1994) quali: ascetica dell'apostolato moderno, morale generale e professionale, medicina e chirurgia, organizzazione ospedaliera, pedagogia e psicologia del malato, assistenza di base e specialistica, formazione delle allieve infermiere e delle infermiere, relazione interprofessionale (tra infermiere religiose e laiche, tra medico e infermiere). Si è inoltre aggiunto un altro raggruppamento denominato "altro" dove inserire gli articoli riguardanti altri professionisti come medici e dietiste. Gli argomenti sono stati aggregati in relazione alla concezione di formazione professionale di Emilia Vinante. Vinante (1954) sostiene che il nucleo centrale della personalità 
dell'infermiera è rappresentato dalla formazione eticoprofessionale, che è immutabile nel tempo, mentre la parte corticale cioè culturale pur essendo essenziale è mutevole e si adatta al progresso delle scienze mediche e delle tecniche infermieristiche.

Nellla figura 1 è riportata la distribuzione degli argomenti secondo la suddivisione sopra riportata in valore percentuale.

Nella figura 2 si analizzano i dati aggregati, cioè il numero degli articoli dedicati alla formazione eticoprofessionale che comprende ascetica dell'apostolato moderno, morale generale e professionale e la parte culturale che comprende medicina e chirurgia, organizzazione ospedaliera, pedagogia e psicologia del malato, assistenza di base e specialistica, formazione delle allieve infermiere e delle infermiere, relazione interprofessionale.

Il totale degli articoli considerati sono 137 , sono stati esclusi dall'analisi gli articoli della categoria altro. Gli articoli che riguardano la formazione etico - professionale sono il $44 \%$ e comprendono gli argomenti riguardanti l'ascetica dell'apostolato moderno, la morale generale e professionale; gli articoli riguardanti la parte culturale sono il 56\%, comprendono gli argomenti di

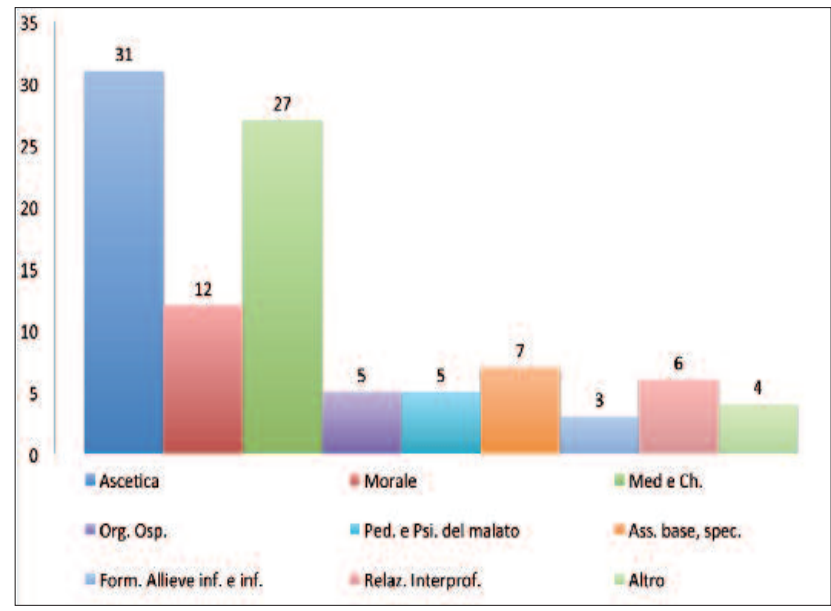

Figura 1: Percentuale di articoli “Quaderni F.I.R.O” suddivisi per argomenti

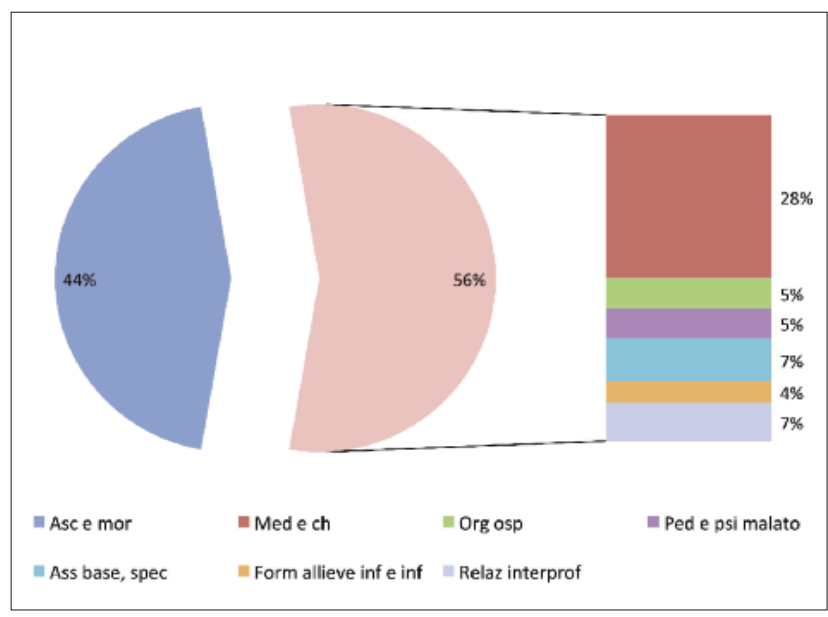

Figura 2: Distribuzione percentuale di articoli aggregati in aree: eticaprofessionale e preparazione culturale. medicina e chirurgia, di organizzazione ospedaliera, pedagogia e psicologia del malato, assistenza di base e specialistica, formazione delle allieve infermiere e delle infermiere, relazione interprofessionale.

\section{DISCUSSIONE}

Obiettivo n.1 - il contributo delle congregazioni religiose rispetto alle principali aree professionali: assistenza diretta, organizzazione/amministrazione, formazione.

L'interpretazione dei dati che riguardano le aree professionali dell'assistenza diretta, dell'organizzazione/amministrazione e della formazione, si presta a essere considerata in modo unitario. La figura professionale che più esprime l'attività della suora in ospedale è la caposala, l'osservazione di questa figura ci offre la possibilità di sintesi dell'evoluzione nelle diverse aree professionali. La suora entra in ospedale come caposala, si occupa della formazione delle allieve e del personale infermieristico e incide nell'assistenza diretta attraverso la formazione morale e tecnica del personale e il controllo dell'assistenza infermieristica erogata. Occorre spiegare perché le amministrazioni ospedaliere richiedevano con insistenza le suore. Scopo delle congregazioni religiose, era di rispondere alle urgenze sociali del tempo, tra cui, le condizioni deplorevoli in ospedale, il personale poco preparato da un punto di vista tecnico, morale e professionale. Si verificavano con una certa frequenza situazioni di incuria e maltrattamenti nei confronti dei pazienti. La suora religiosa era richiesta dall'amministrazione ospedaliera più come sorvegliante del materiale delle cucine, del personale, del movimento-malati, che per favorire il miglioramento della qualità dell'assistenza.

Con la figura della caposala, le congregazioni religiose, riescono a conciliare le esigenze delle amministrazioni ospedaliere con le proprie, cioè di fornire un'assistenza caritatevole nei confronti dei malati. La loro importante opera di educazione morale al personale e al malato è avvenuta attraverso uno studio per definire il comportamento morale, si veda ad esempio nella figura 2, la percentuale degli articoli dei "Quaderni FIRO al 44\% inerenti all'area etico - professionale. Le amministrazioni riconoscono la superiorità gerarchica della suora sulle laiche, chiedono alle suore di assumere posti direttivi e alle laiche invece il servizio di assistenza diretta (Vinante, 1946). Le suore entrano negli ospedali con il ruolo di caposala, in seguito sono richieste anche per l'assistenza diretta (Cozzani, 1957).

Suor Vinante attraverso le caposala religiose si propone di ottenere una coerenza etica e tecnica delle infermiere rispetto agli insegnamenti della scuola convitto. 
Nel mondo contemporaneo il gap tra teoria e pratica costituisce il problema maggiore per l'apprendimento professionale degli studenti, la letteratura riporta che il coinvolgimento del mondo accademico con quello sanitario, mediante l'applicazione di modelli educativi, favorisce l'apprendimento dello studente e una migliore pratica infermieristica. Nell'epoca di suor Vinante la filosofia di formazione delle allieve, del personale e dell'assistenza dell'ammalato era condivisa dalle suore che si occupavano di formazione, di organizzazione e di assistenza diretta.

La formazione "etica" avviene mediante l'imitazione di Cristo e attraverso la relazione educativa che inizia nella scuola convitto e continua anche dopo il diploma: "L'esperienza fatta nel nostro grande ospedale fa rilevare sempre più come sia assolutamente indispensabile una severa, diligente, accurata e solerte formazione delle infermiere durante il biennio di scuola, continuandola possibilmente una volta che le giovani diplomate passano in servizio ad altri reparti". Secondo Vinante (Negri 2007) il lavoro educativo avviene nella relazione: "Non si può fare del bene se non amando (...) non dimenticando mai che ciascuna (allieva) ha la sua personalità e i suoi diritti."

La vita delle allieve è scandita dalle preghiere, dallo studio, anche i momenti di ricreazione sono l'occasione per le allieve di coltivare il loro spirito, in compagnia della direttrice, vice-direttrice, diplomate addette alla scuola e alcune suore caposala.

Obiettivo n. 2 - il contributo che le congregazioni religiose hanno dato allo sviluppo degli attributi distintivi della professione infermieristica, nell'ottica sociologica

Si analizzano ora le fonti in riferimento agli attributi di una professione individuati da Greenwood, (1980).

Il riconoscimento della professione infermieristica è presente in più relazioni. Suor Lauriola nel 1965, afferma: "Infatti, oggi più complesse sono le funzioni dell'infermiera professionale e anche se per molti rimane ancora una esecutrice di ordini, essa in realtà svolge compiti di alta responsabilità in campi diversi".

Analizzando il corpo sistematico di teoria, possiamo affermare che le attività promosse dalla FIRO e l'impegno nella formazione professionale di suor Emilia Vinante hanno favorito il consolidamento e l'acquisizione di un corpo sistematico di conoscenze.

Suor Emilia Vinante (1954), a tal riguardo afferma: "L'attività professionale richiede un insieme di cos̀̀ numerose cognizioni e di tanta larga esperienza da costituire una vera e propria attività professionale... L'abilità professionale presuppone dunque scienza e tecnica ed anche richiede una sicura esperienza che possa guidare chi assiste nei molti difficili casi della vita quotidiana. L'acquisto delle capacità tecniche professionali è frutto di un lungo e paziente lavoro durante gli anni scolastici mediante un tirocinio pratico condotto con seri criteri didattici assistenziali, tali da permettere all'allieva del tutto inesperta della materia di assimilare i concetti e le nozioni scientifiche, che dovranno poi guidarla nella sua futura attività infermieristica.". Il tirocinio pratico secondo Vinante condotto con seri criteri didattici, non è semplicemente l'apprendimento del saper fare, ma del sapere, tale da permettere all'allieva di assimilare i concetti e le nozioni scientifiche.

La definizione dei concetti fondamentali del comportamento professionale: il concetto di uomo, ambiente, salute/malattia e assistenza infermieristica, ha rappresentato il primo passo nella definizione della Disciplina Infermieristica (Manzoni, 2005).

Nei documenti sono descritti i concetti di uomo, salute/malattia e di assistenza infermieristica. Suor Lauriola (1965) definisce il concetto di uomo: "Quella persona umana che va considerata non solo nelle sue funzioni somatiche ma anche in quelle psichiche, poiché è risaputo che un'alterazione organica localizzata, comporta una sofferenza psichica che investe tutta la personalità dell'individuo", e il concetto di assistenza infermieristica: "La storia dell'assistenza noi la possiamo identificare con la storia stessa dell'umanità proprio perché soggetta alle infermità e alla morte ha istintivamente dal suo nascere provveduto a difendersi dall'una e dall'altra. Una breve rassegna storica dalle antiche civiltà ai nostri tempi, ci direbbe la perennità del problema assistenziale, ci mostrerebbe tutti gli sforzi che in ogni tempo l'uomo ha compiuto per difendere quel gran bene e quel gran dono che è la salute, anche quando la medicina era assolutamente empirica e primordiale.”.

Suor Carissoni (1972), descrive il concetto di salutemalattia: "Chiunque si trovi in uno stato di sofferenza non tarda ad assumere atteggiamenti e modi di agire molto simili a quelli che assume il bambino o una persona anziana. Una persona in stato di sofferenza scivola nella regressione mentale e affettiva... La persona malata che soffre tende istintivamente a fare ciò che fa il bambino: attira lì attenzione su di sé: e se la sua infermiera non la guarda come lei desidera, farà qualcosa per sollecitarne le premure: accuserà un mal di testa o disturbi di qualsiasi altro genere".

Molto interessante l'approccio empatico al malato descritto da Suor Carissoni (1072): "Se una infermiera non comprende tutte queste reazioni" (si riferisce a regressione, depressione, sensi di colpa) da parte della persona sofferente finisce col farsi giocare dalla stessa persona che deve curare. Deve assumere e mantenere sempre un atteggiamento neutrale dal punto di vista affettivo e lasciare parlare. Deve ascoltare. Lasciare che queste persone scarichino la loro aggressività, tutti i sentimenti negativi in modo da permettere loro meglio coscienza del loro problema”.

Dai risultati della ricerca, rispetto all'autorità profes- 
sionale, emerge che la superiora esercitava una leadership nei confronti delle suore, delle infermiere e delle allieve aveva un campo d'azione proprio, autonomo rispetto alle amministrazioni ospedaliere, difeso con attenzione dalla FIRO

Nelle relazioni della FIRO, (1957, 1963), sono descritti i punti necessari per la revisione delle convenzioni con le amministrazioni ospedaliere, emerge con chiarezza l'indicazione che il personale religioso mantenga la superiorità gerarchica sul personale laico e che l'amministrazione del personale religioso sia esercitato esclusivamente dalla superiora e non dall'amministrazione dell'ospedale.

Troviamo ampia documentazione dell'impegno delle suore a implementare il codice regolativo dell'etica sia nelle relazioni ai corsi convegni sia negli articoli dei "Quaderni FIRO". Suor Vinante (1949), afferma "Sia laica o religiosa, l'infermiera ha una sua netta figura morale, una figura di forte rilievo sullo sfondo sociale, essa svolgendo le sue mansioni assolve una missione di carità e d'amore che la rende una creatura moralmente superiore che offre alla società con tutta l'abnegazione possibile la sua attività ed il suo cuore".

Il comportamento morale è insegnato mediante la preghiera, nella preghiera delle allieve infermiere, l'allieva chiede a Dio serenità, pacatezza, padronanza di noi stesse, dolcezza e affabilità di sguardi di modi e di parole, purezza di contegno e di pensiero, sorriso buono sul labbro e sul cuore, mani gentili, cuori generosi, anime pazienti.

Per quanto riguarda la sanzioni della comunità, FIRO si è impegnata per il riconoscimento dell'utilità sociale da parte della popolazione rispetto alla professione infermieristica

Rispetto alla cultura professionale l'aspetto più indicativo era che le suore per la loro opera ricevevano spesso riconoscimenti ufficiali, medaglie dalle amministrazioni ospedaliere (Baldinucci, 1964).

\section{CONCLUSIONI}

Nell'affrontare il presente lavoro ci si è imbattuti nella nascita delle congregazioni religiose, nella storia di donne religiose che hanno dimostrato di essere all'altezza delle richieste socio-assistenziali del XIX secolo. Si è preso come esempio storico le Suore di Maria Bambina in quanto è stata una delle congregazioni lombarde e italiane di maggior rilievo sia per la diffusione e il numero di membri, sia per il contributo dato allo sviluppo dell'assistenza infermieristica in ospedale e alla formazione delle allieve nelle scuole convitto per infermiere. Inizialmente il lavoro di ricerca aveva come obiettivo di evidenziare il contributo che le Suore di Maria Bambina hanno apportato nelle aree professionali, e allo sviluppo degli attri- buti distintivi della professione infermieristica nell'ottica sociologica. Il reperimento di fonti della FIRO ci ha consentito di ampliare ed estendere l'argomento al contributo delle congregazioni religiose.

Alla luce dei risultati della ricerca si può affermare che le congregazioni religiose hanno operato nell'ambito della professione infermieristica diventando una forza sociale in grado di incidere in modo determinante sullo sviluppo culturale e professionale degli infermieri.

Si sono adoperate per istituire e dirigere le scuole convitto per infermiere, ritenendo che la preparazione di personale qualificato fosse un dovere della comunità, per intrattenere rapporti con le Associazioni, con il Ministero, con il Collegio delle infermiere professionali e fornire un valido aiuto nell'interpretare i bisogni della popolazione. Le Congregazioni religiose hanno favorito il riconoscimento dell'attività professionale dell'infermiere e proposto soluzioni di politica professionale.

La presente ricerca storica vuole dare un contributo alla storiografia della donna religiosa del XIX secolo e si propone di stimolare altre ricerche sulla storia dell'assistenza infermieristica affinché si forniscano nuovi elementi conoscitivi utili al gruppo professionale.

\section{BIBLIOGRAFIA}

Beretta, O. (1984) Il contributo di suor Emilia Vinante quale spinta evolutiva nella formazione dell'infermiere professionale [tesi]. Roma:tesi di specializzazione presso la scuola speciale per dirigenti dell'assistenza infermieristica, Università Cattolica del Sacro Cuore.

Bartoloni S. (2007) Per le strade del mondo. Laiche e religiose fra otto e novecento. Bologna: società editrice il Mulino.

Chabod, F., (2006). Lezioni di metodo storico. Roma-Bari: Laterza.

Carraro, M., \& Mascotti A. (1987) Listituto delle sante Bartolomea Capitanio e Vincenza Gerosa. Milano:Arti grafiche De Carli vol I-II.

Colombo, A. (2004) Congregazioni religiose e sviluppo in Lombardia tra Otto e Novecento. Il caso delle Suore di Maria Bambina. Milano: Vita e Pensiero.

Cossetta, A.B. (1994) Professione infermiere organizzazione normativa etica Torino: UTET.

Greenwood, E. (1980). Contributi alla teoria generale della professionalità. In: Prandstraller GP Sociologia delle professioni. Roma: Città Nuova Editrice, 103118.

Manzoni, E. (2005) Storia e filosofia dell'assistenza infermieristica. Milano: Masson.

Negri, M. (2005) Il corso per aspiranti direttrici di scuole infermiere, ospedale Niguarda, 1953 - 1965 [tesi]. Monza: Università degli Studi di Milano-Bicocca.

Negri, M. (2007) Suor Emilia Vinante, Direttrice di Scuola Infermiere a Milano. Journal of medicine and the person, 5(1):28-30

Rocca, G. (1992) Donne religiose citato in Colombo A. (2004) Congregazioni religiose e sviluppo in Lombardia tra Otto e 
Novecento. Il caso delle Suore di Maria Bambina. Milano: Vita e Pensiero

Sironi, C. (1992) Storia dell'assistenza infermieristica Roma: La Nuova Italia Scientifica.

\section{BIBLIOGRAFIA DELLE FONTI STORICHE}

Carissoni ML. (1972) La funzione della suora infermiera in comunità. Quaderni FIRO 5:32-37. in AG SdC, cartella 493, fascicolo E.

Editorale: $I^{\circ}$ Convegno nazionale della FIRO Roma 24-28 aprile 1957. Quaderni FIRO 3:1-2. in AG SdC.

Lauriola E. (1965) Evoluzione delle professioni sanitarie ausiliarie in rapporto alle strutture sanitario-assistenziali del nostro paese. Quaderni FIRO 3:32-42. in AG SdC.

Lettera Circolare, di suor Cozzani alle reverende Madri Generali, 15 dicembre 1960, in AG SdC, cartella 420, fascicolo Q.

Lettera - FIRO- Suor Cozzani, Roma alla reverenda Madre, Roma 5 agosto 1957, in AG SdC, cartella 420, fascicolo P.

Lettera - S. Congregazione dei Religiosi a Suor Costantina Baldinucci, Roma 5 aprile 1959, in AG SdC, cartella 420, fascicolo P.

Lettera a Suor Cozzani, 8 agosto 1957, in AG SdC, cartella 420, fascicolo P.

Lettera Circolare Riservata, FIRO anno 1957-58? in AG SdC, cartella 421, fascicolo E.

Lettera Circolare, di suor Costantina Baldinucci, Roma 29 gennaio 1964, in AG SdC, cartella 421, fascicolo A.

Lettera Circolare, di suor Cozzani alle reverende superiore degli ospedali d'Italia, Roma 1 agosto 1946, in AG SdC, cartella 421, fascicolo A.

Lettera Circolare, di suor Cozzani alle reverende superiore degli ospedali d'Italia, Roma 1 agosto 1964, in AG SdC, cartella 421, fascicolo B.

Lettera Circolare, di suor Cozzani, in AG SdC, cartella 421, fascicolo A.

Lettera Circolare, di suor Cozzani, Roma, 12 agosto 1967, in AG SdC, cartella 421, fascicolo C.

Lettera Circolare, di suor Cozzani, Roma, 8 giugno 1966, in AG SdC, cartella 421, fascicolo C.

Lettera della segreteria interdiocesana Lombardia delle federazioni tra le religiose d'Italia, in AG SdC, cartella 174, fascicolo J.

Lettera di Carmen Campi alla Reverenda Madre Superiora Generale, Milano 16 gennaio 1948, in AG SdC, Milano ospedale Maggiore decreti e regolamenti.

Lettera di Maria Senni Roma 16 agosto 1946. In AG SdC, cartella 174 , fascicolo J.

Lettera di Maria Senni Roma 2 agosto 1946. in AG SdC, cartella 174 , fascicolo J.

Lettera di Maria Sforza alla madre generale delle Suore di Maria Bambina, Milano 15 aprile 1931, in allegato la relazione delle scuole professionali cattoliche inglesi, in AG SdC, Milano ospedale Maggiore decreti e regolamenti.

Lettera di suor Baldinucci a Suor Cozzani, 6 III 1963, in AG SdC, cartella 420, fascicolo Q.

Lettera di suor Torchio alla reverenda Madre, Roma, 15 febbraio 1968, in AG SdC, cartella 421, fascicolo D.

Lettera S. Congregazione dei Religiosi alla superiora generale Madre Angiolina Reali, Roma, 10 novembre 1949, in AG SdC, cartella 241, fascicolo B.

Lettera promemoria, S. Congregazione dei Religiosi, 22 gennaio 1968, in AG SdC, cartella 421, fascicolo D.

Padre Molinari F. (1956) Editoriale: I nostri quaderni. Quaderni FIRO 1:1-2. in AG SdC.

Padre Molinari F. (1956) Editoriale: I nostri quaderni. Quaderni FIRO 2:1-3. in AG SdC.

Preghiera delle allieve infermiere della scuola convitto infermiere degli Istituti Ospitalieri di Milano in AG SdC, Milano ospedale Maggiore decreti e regolamenti.

Programma corso d'aggiornamento - FIRO, Milano 15 gennaio-18 marzo 1966, in AG SdC, cartella 421, fascicolo $\mathrm{H}$.

Programma I Convegno Nazionale - FIRO, Roma 24-28 aprile 1957, in AG SdC, cartella 420, fascicolo P.

Quaderni FIRO "Infirmus eram et visitastis me" anno $1^{\circ}$ N.1 gennaio febbraio marzo 1956, in AG SdC.

Quaderni FIRO "Infirmus eram et visitastis me" anno $1^{\circ}$ N.2 aprile maggio giugno 1956, in AG SdC.

Quaderni FIRO "Infirmus eram et visitastis me" anno $1^{\circ} \mathrm{N} .3$ luglio agosto settembre 1956, in AG SdC.

Quaderni FIRO “Infirmus eram et visitastis me" anno $1^{\circ}$ N.4 ottobre novembre dicembre1956, in AG SdC.

Quaderni FIRO "Infirmus eram et visitastis me" anno $2^{\circ}$ N.2 aprile maggio giugno 1957, in AG SdC.

Quaderni FIRO "Infirmus eram et visitastis me" anno $2^{\circ}$ N.3 luglio agosto settembre 1957, I. Convegno nazionale della FIRO, in AG SdC.

Quaderni FIRO "Infirmus eram et visitastis me" anno $2^{\circ}$ N.4 ottobre novembre dicembre1957, in AG SdC.

Quaderni FIRO "Infirmus eram et visitastis me" anno $3^{\circ}$ N.1 gennaio febbraio marzo 1958, in AG SdC.

Quaderni FIRO “Infirmus eram et visitastis me" anno $3^{\circ}$ N.2 aprile maggio giugno 1958, in AG SdC.

Quaderni FIRO "Infirmus eram et visitastis me" anno $4^{\circ} \mathrm{N} .1$ gennaio febbraio marzo 1959, in AG SdC.

Quaderni FIRO “Infirmus eram et visitastis me" anno $4^{\circ}$ N.2 aprile maggio giugno 1959, in AG SdC.

Quaderni FIRO "Infirmus eram et visitastis me" anno $4^{\circ}$ N.4 ottobre novembre dicembre1959, in AG SdC.

Quaderni FIRO “Infirmus eram et visitastis me" anno $5^{\circ}$ N.1 gennaio febbraio marzo 1960, in AG SdC.

Quaderni FIRO "Infirmus eram et visitastis me" anno $5^{\circ}$ N.2 aprile maggio giugno 1960, "Atti del convegno nazionale F.I.RO, in AG SdC.

"Quaderni FIRO delle religiose ospedaliere" anno X Num. 2 marzo aprile $1965^{\prime \prime}$, in AG SdC.

Quaderni FIRO delle religiose ospedaliere” anno X Num. 3 maggio giugno 1965", in AG SdC.

Quaderni FIRO delle religiose ospedaliere” anno X Num. 45 luglio-agosto sett.-ottobre 1965, in AG SdC.

"Quaderni FIRO delle religiose ospedaliere" anno XI Num. 1 gennaio febbraio 1966, in AG SdC.

"Quaderni FIRO delle religiose ospedaliere" anno XI Num. 2 marzo aprile 1966, in AG SdC.

"Quaderni FIRO delle religiose ospedaliere" anno XII Num. 1 
gennaio febbraio 1967, in AG SdC.

"Quaderni FIRO delle religiose ospedaliere" anno XII Num. 4 luglio agosto 1967, in AG SdC.

"Quaderni FIRO delle religiose ospedaliere" anno XII Num. 5-6 agosto settembre1967, in AG SdC.

"Quaderni FIRO delle religiose ospedaliere" anno XIII Num. 2 marzo aprile 1968, in AG SdC.

Relazione annuale FIRO, in AG SdC, cartella 421, fascicolo A.

Relazione dott Germano Sollazzo: "La scuola convitto professionale per infermiere degli Istituti ospitali eri di Milano nel suo primo venticinquennio di vita: 19311956". In AG SdC, Milano ospedale Maggiore decreti e regolamenti.

Relazione di Madre Baldinucci presidente FIRO - 22 aprile 1965 in AG SdC, cartella 421, fascicolo B.

Relazione di Maria Sforza: "La scuola professionale convitto infermiere per religiose e laiche degli Istituti Ospitalieri di Milano nel suo primo quinquennio di funzionamento". In AG SdC, Milano ospedale Maggiore decreti e regolamenti.

Relazione di Suor Emilia Vinante: "L'assistenza ospedaliera in Italia" 5 novembre 1949, in AG SdC, cartella 241, fascicolo B.

Relazione di Suor Emilia Vinante: "Scienza e abilità professionale" in AG SdC, cartella 174, fascicolo J.

Relazione di Suor Emilia Vinante, 18 agosto 1946: "La suora e l'infermiera laica nella fusione del lavoro" in AG SdC, cartella 174 , fascicolo J.

Relazione FIRO: "Elenco delle Scuole Convitto per Infermiere Professionali, Assistenti Sanitarie Visitatrici, Vigilatrici d'Infanzia e Assistenti Dietiste, dirette da Religiose, 30 gennaio 1963, in AG SdC, cartella 420, fascicolo Q.

Relazione, 1957,"Punti di particolare interesse che si sottopongono all'attenzione delle congregazioni religiose femminili per l'elaborazione delle singole convenzioni con gli enti ospedalieri”, in AG SdC, cartella 420, fascicolo $\mathrm{Q}$.

Relazione, 1963, "Punti di particolare interesse che si sottopongono all'attenzione delle congregazioni religiose femminili per l'elaborazione delle singole convenzioni con gli enti ospedalieri”, in AG SdC, cartella 420, fascicolo Q, in AG SdC.

Relazione: "Perfezionamento del personale didattico delle scuole convitto", in AG SdC, cartella 174, fascicolo K.

Vinante E. (1952).Note di tecnica e pratica per l'assistenza infermieristica medico-chirurgica. Milano: tipografia Naccari.: Andamento nelle testate censite delle categorie Ricerca e Teoria dal 1997 al 2009

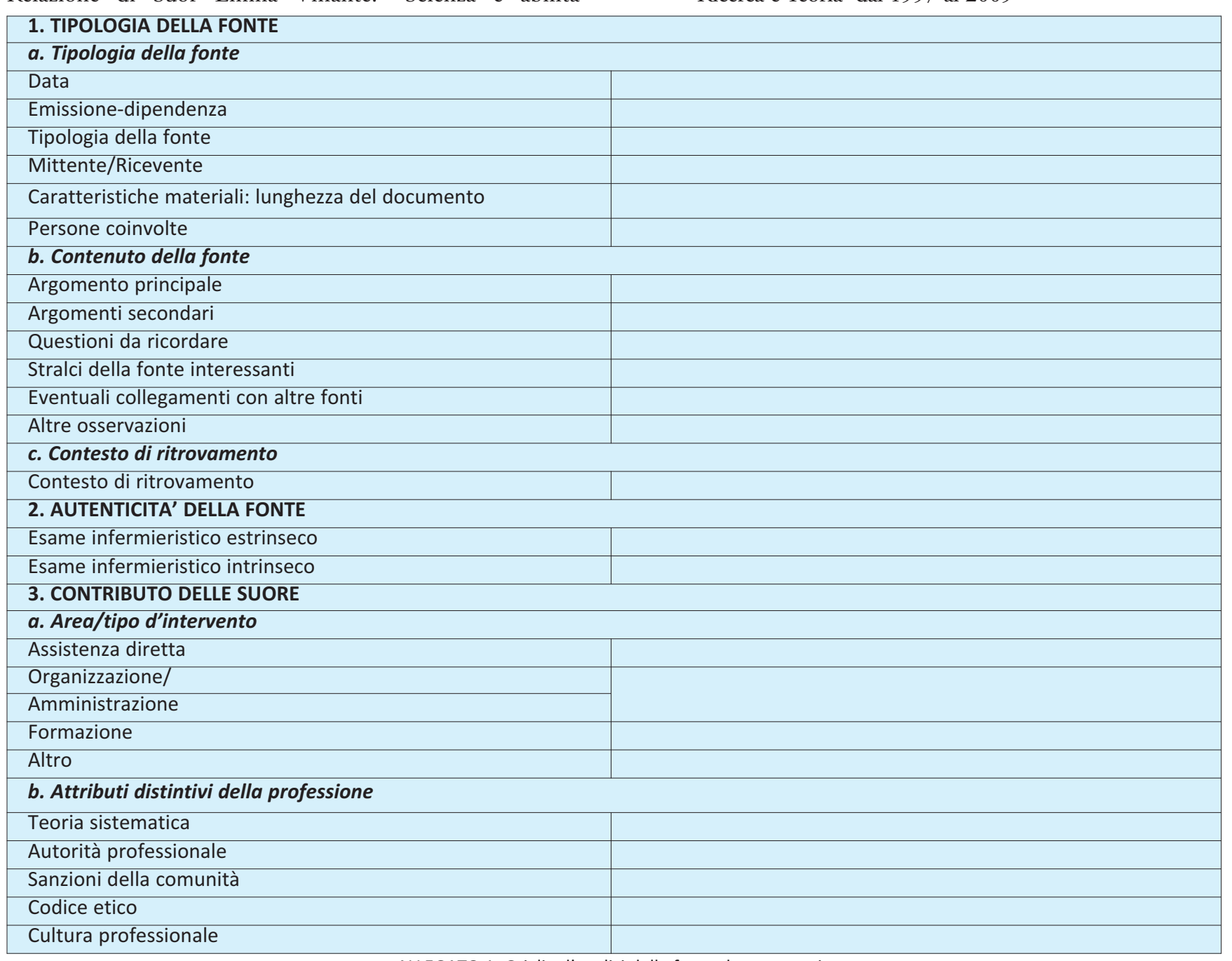

